\title{
Estrés Postraumático en Niños y Adolescentes Abusados Sexualmente
}

\author{
PAULINA RINCÓN G.*1, FÉLIX COVA S.*1, PATRICIA BUSTOS T.*2, \\ JAIME AEDO S. ${ }^{* 3}$, MARIO VALDIVIA P. ${ }^{4}$ \\ 1. Psicólogo(a), Dr(a). en Psicología, Dpto. de Psicología, Facultad de Ciencias Sociales, Universidad de Concepción. \\ 2. Trabajadora Social, Magíster en Psicología mención Salud. \\ 3. Psicólogo, Escuela de Psicología, Universidad Santo Tomás Sede Los Ángeles. \\ 4. Médico Psiquiatra, Dpto. de Psiquiatría, Facultad de Medicina, Universidad de Concepción. \\ Departamento de Psicología, Facultad de Ciencias Sociales, Universidad de Concepción.
}

\begin{abstract}
Post-Traumatic Stress Disorder among Sexually Abused Children and Adolescents

Introduction: The development of Post-Traumatic Stress Disorder (PTSD) is a frequently observed effect of sexual abuse among children and adolescents. Objectives: This study focused on the prevalence of PTSD among children and adolescents who were victims of sexual abuse. Method: A sample of 75 sexually abused children and adolescentes seeking outpatient care were evaluated utilizing a Children's Post Traumatic Stress Scale (CPSS). Results: $21.3 \%$ met the criteria for PTSD, an additional $16 \%$ met the criteria for a SubSyndrome. Conclusions: These data confirm the importance of focusing on post-traumatic symptomatology on sexually abused children and adolescents. Equally important is the finding that the majority of the children in the sample did not show symptomatology at the time of the study.
\end{abstract}

(Key words: Sexual abuse, Post-Traumatic Stress Disorder, PTSD, children, adolescent).

Rev Chil Pediatr 2010; 81 (3): 234-240

\section{RESUMEN}

Introducción: El desarrollo de sintomatología postraumática es uno de los efectos del abuso sexual de niños y adolescentes que se observa con mayor frecuencia. Objetivos: En el presente estudio se evaluó la prevalencia de trastorno de estrés postraumático y del denominado subsíndrome de estrés postraumático en niños y adolescentes víctimas de abusos sexuales. Método: La muestra estuvo constituida por 75 niños y adolescentes sexualmente abusados consultantes de centros de atención quienes fueron evaluados con la Escala Infantil de Síntomas del Trastorno de Estrés Postraumático (CPSS). Resultados: Un 21,3\% cumplió con los criterios para el diagnóstico de Trastorno de Estrés Postraumático, y un 16\% adicional cumplió con los criterios de

Trabajo recibido el 07 de abril de 2010, devuelto para corregir el 26 de abril de 2010, segunda versión el 27 de abril de 2010 , aceptado para publicación el 29 de abril de 2010.

Correspondencia a:

Paulina Rincón G.

E-mail: princon@udec.cl 
subsíndrome de Estrés Postraumático. Conclusiones: Estos datos confirman la importancia de considerar la sintomatología postraumática como un foco de evaluación y tratamiento en niños y adolescentes sexualmente abusados. Sin embargo, es de importancia destacar que la mayoría de los niños y adolescentes evaluados no informó presentar sintomatología postraumática acusada al momento del estudio.

(Palabras clave: Trastorno de estrés postraumático, niñez, adolescencia, abuso sexual).

Rev Chil Pediatr 2010; 81 (3): 234-240

\section{Introducción}

Los contextos y formas en que pueden darse experiencias calificables como abuso sexual de niños y adolescentes (en adelante, se utilizará el concepto de "abuso sexual infantil" englobando tanto el abuso sexual de niños como el de adolescentes) son muy diferentes. En términos generales, el abuso sexual infantil constituye un significativo factor de riesgo de desarrollo de psicopatología, no sólo en momentos cercanos a la experiencia abusiva, sino distanciados de ésta temporalmente ${ }^{1-3}$.

Si bien legalmente se distingue entre abuso sexual y violación, en el contexto del presente artículo se utiliza el concepto de abuso sexual infantil en un sentido amplio, que abarca las distintas formas en que niños y adolescentes son implicados en actividades sexuales por personas que les superan de modo significativo en edad ${ }^{4}$.

Dado que la experiencia abusiva puede darse de formas muy distintas, es complejo establecer en términos absolutos las consecuencias psicológicas que genera la experiencia de abuso sexual. No existe un síndrome específico post abuso sexual; el impacto potencial del abuso sexual puede abarcar muy distintos ámbitos del comportamiento y desarrollo ${ }^{5}$. Las consecuencias psicológicas del abuso sexual se encuentran relacionadas con una serie de variables que suelen interrelacionarse de modo complejo. Entre otras, han sido destacadas la frecuencia y severidad de la experiencia abusiva; el tipo de vínculo con el agresor; la presencia de violencia directa durante el abuso; el grado de culpa y victimización del niño/a o adolescente por parte de la familia o referentes significativos; la confianza demostrada hacia él; las estrategias de afrontamiento que tenga la víctima y las consecuencias derivadas de la develación ${ }^{6}$.

$\mathrm{Al}$ respecto, es particularmente importante considerar el distinto carácter de las experiencias abusivas que se observan con mayor frecuencia en los estudios de población general, y aquellas que se observan en muestras clínicas. En los estudios de población general, la mayoría de las personas que refieren haber vivido experiencias abusivas describen episodios únicos y de muy distintos niveles de gravedad; en cambio, en centros clínicos, la mayoría de las experiencias de abuso sexual corresponden a situaciones abusivas repetitivas, con alta frecuencia correspondientes a abusos sexuales intrafamiliares ${ }^{7}$. Otra diferencia importante es que en esta segunda situación suele ser más frecuente también la existencia de otras condiciones de adversidad para el desarrollo del niño o niña, lo que hace particularmente complejo determinar qué factores son los principales responsables de los efectos negativos observados en el desarrollo de quienes se han visto expuesto a ellos ${ }^{8,9}$.

Entre las consecuencias psicológicas del abuso sexual infantil que se ha observado con mayor frecuencia y especificidad están el desarrollo de comportamientos sexualizados inapropiados para la edad y la presencia de estrés postraumático, foco de interés del presente estudio $^{5,10}$.

La prevalencia observada de trastorno de estrés postraumático (TEP) en víctimas de abuso sexual infantil difiere entre los estudios de modo notorio según el tipo de población en que se ha realizado. McLeer et $\mathrm{al}^{11}$, estudian un grupo de niños sexualmente abusados de 6 a16 años en un período cercano (uno a dos meses) al descubrimiento del abuso, encontrando una prevalencia de TEP de 36,3\%; 65\% 
de los niños presentaban uno o más síntomas de reexperimentación; $43,7 \%$ presentaba tres o más conductas de evitación; un $57,5 \%$ presentaba dos o más síntomas de hiperactivación autonómica. En muestras clínicas se observan tasas más altas. La misma autora, en estudios realizados en muestras clínicas, observó tasas de más de $40 \%$ de TEP. Tasas aún más altas fueron observadas en una muestra clínica de niños abusados de Londres: $71 \%$ de TEP ${ }^{12}$.

Dada la alta frecuencia tanto de sintomatología postraumática y de trastorno de estrés postraumático propiamente tal en niños y adolescentes sexualmente abusados, y el grado de impedimento y persistencia que puede tener este patrón psicopatológico, se ha planteado la utilidad de evaluar su presencia regularmente en las víctimas de abuso sexual infantil.

En Chile, el estrés postraumático en niños y adolescentes abusados ha sido poco investigado. El presente estudio tiene por objetivo estimar la presencia de sintomatología y trastorno de estrés postraumático, en niños y adolescentes sexualmente abusados, que estaban siendo evaluados o tratados por esta situación.

\section{Método}

\section{Participantes}

La muestra estuvo constituida por 75 niños (as) y adolescentes víctimas de violencia sexual, en peritaje o tratamiento, tanto en Centros de Diagnóstico Ambulatorio (DAM) como en Programas Especializados en Maltrato, y en ciertos hogares para niños en esta situación. Los criterios de inclusión fueron: a) tener entre 8 y 18 años y b) haber sido víctima de violencia sexual, con presencia de informe sexológico forense que indicara lesiones atribuibles a violencia sexual o peritaje de veracidad de testimonio que calificara el relato como creíble. Fue criterio de exclusión presentar algún grado de retardo mental o analfabetismo. $\mathrm{La}$ evaluación se realizó entre Enero y Junio de 2008. La edad promedio de la muestra fue de 12,15 años $(D E=2,8)$; el 25,3\% eran hombres y el $74,7 \%$, mujeres.

Legalmente se distingue entre abuso sexual y violación. El 64\% de los participantes había vivido abuso sexual y el 36\%, violación. El agresor se encontraba con mayor frecuencia en el núcleo intrafamiliar (63\%), seguido del agresor sexual extrafamiliar conocido (25\%) $\mathrm{y}$, por último, el agresor extrafamiliar desconocido $(12 \%)$.

Un $63 \%$ de los participantes contaba con pruebas periciales relativas a la constatación de lesiones y veracidad de testimonio de manera conjunta, un $13 \%$ contaba únicamente con pruebas periciales relativas a la constatación de lesiones, un $17 \%$ contaba sólo con pruebas de veracidad de testimonio y un $7 \%$ no contaba con ninguna prueba pericial. Esto último se debe a que la historia de los niños que ingresaron por abuso sexual correspondía a tocaciones, por lo cual los profesionales no tenían mayor información pericial, sino sólo la correspondiente a cambios evidentes de conducta y al relato de los niños (as) o adolescentes, otorgando éstos credibilidad al relato (tabla 1).

\section{Variables e Instrumentos}

a) Antecedentes sociodemográficos e historia de violencia sexual de los participantes. Esta información fue obtenida de los registros existentes en los Centros.

b) Estrés postraumático. Esta variable fue evaluada a través de la Escala Infantil de Sintomas del Trastorno de Estrés Postraumático (CPSS). La CPSS es un instrumento desarrollado para evaluar la presencia de síntomas del Trastorno de Estrés Postraumático en niños y niñas de 8 a 18 años de edad con una historia conocida de trauma. La Escala está basada en los criterios diagnósticos del DSM-IV y se compone de 17 ítems con respuesta tipo Likert referidos a la frecuencia de manifestación de síntomas de este trastorno ${ }^{13}$. La CPSS se compone de 3 subescalas: Reexperimentación (5 ítems), Evitación (7 ítems) y Aumento de la Activación (5 ítems). Las propiedades psicométricas de la versión original de la CPSS indican niveles aceptables de confiabilidad por consistencia interna y test-retest. La CPSS ha mostrado una correlación de $r=0,75$ con la Escala para Estrés Post Traumático en Niños (CPTSD, construida por Frederik et al, en 1985 y basada en los criterios diagnósticos para TEP 
Tabla 1. Características de la violencia sexual en la muestra

\begin{tabular}{|llcc|}
\hline Variable de Historia de Abuso Sexual & Categorías & $\mathbf{n}$ & $\%$ \\
\hline Tipo de agresión sexual & Violación & 27 & 36,0 \\
\hline Tipo de agresor & Abuso sexual & 48 & 64,0 \\
& Intrafamiliar & 47 & 62,7 \\
\hline Recibe tratamiento tras agresión & Extrafamiliar conocido & 19 & 25,3 \\
& Extrafamiliar desconocido & 9 & 12,0 \\
\hline Agresor vive con el niño/a o adolescente & Sí & 56 & 74,7 \\
& No & 19 & 25,3 \\
\hline Pruebas periciales & Sí & 3 & 4,0 \\
& No & 72 & 96,0 \\
\hline Tiempo transcurrido entre agresión y aplicación CPSS & Constatación de lesiones y credibilidad de testimonio & 47 & 62,7 \\
& Constatación de lesiones & 10 & 13,3 \\
\hline & Credibilidad de testimonio & 13 & 17,3 \\
\hline & Sin pruebas periciales & 5 & 6,7 \\
\hline
\end{tabular}

del DSM-III). Las propiedades psicométricas de la escala en la población de este estudio fueron informadas con detalle en una publicación previa $^{14}$. Brevemente, los coeficientes alfa de Cronbach oscilaron entre 0,78 y 0,92 , siendo muy similares a los señalados respecto del instrumento original ${ }^{13}$.

\section{Resultados}

La Escala CPSS no considera puntajes de corte para establecer diagnóstico de trastorno de estrés postraumático ${ }^{13,14}$. Se utilizaron los criterios DSM-IV-TR que establecen la presencia de al menos 1 síntoma de reexperimentación, 3 de evitación y 2 de aumento de activación para considerar que una persona presenta el trastorno ${ }^{15}$. Se utilizó un criterio conservador para calificar la presencia de síntomas: el síntoma fue considerado presente sólo cuando las respuestas referían que éste era experimentado en las dos últimas semanas "entre cuatro y ocho veces" o "nueve veces o más". Si el síntoma era informado como ausente o presente "sólo una vez" o "entre dos y tres veces" en las últimas dos semanas, se estimó que no estaba presente. Se optó por este criterio dado que el instrumento no permite evaluar el grado de discapacidad asociada a los síntomas, y por tratarse de un criterio consistente con los utilizados en otras escalas desarrolladas para el diagnóstico de $\mathrm{TEP}^{16}$.

La tabla 2 muestra las prevalencias obtenidas. Un $21,3 \%$ de los participantes presenta trastorno de estrés postraumático y un $16 \%$

Tabla 2. Diagnóstico de trastorno por estrés postraumático o su subsíndrome

\begin{tabular}{|lcc|}
\hline Categoría & n & \% \\
\hline Ausencia TEP & 47 & 62,7 \\
\hline Subsíndrome TEP & 12 & 16,0 \\
\hline Presencia TEP & 16 & 21,3 \\
\hline
\end{tabular}


Tabla 3. Frecuencia de los diferentes síntomas del trastorno de estrés postraumático en las últimas 2 semanas

\begin{tabular}{|c|c|c|c|c|c|}
\hline Síntoma & Nunca & Sólo 1 vez & $\begin{array}{c}2 \text { a } 3 \\
\text { veces }\end{array}$ & $\begin{array}{c}4 \text { a } 8 \\
\text { veces }\end{array}$ & $\begin{array}{c}9 \text { veces o } \\
\text { más }\end{array}$ \\
\hline \multicolumn{6}{|l|}{ Reexperimentación } \\
\hline Recuerdos intrusivos & $17(22,7 \%)$ & $18(24 \%)$ & $21(28 \%)$ & $8(10,7 \%)$ & $11(14,7 \%)$ \\
\hline Sueños intrusivos & $21(28 \%)$ & $20(26,7 \%)$ & $20(26,7 \%)$ & $6(8 \%)$ & $8(10,7 \%)$ \\
\hline "Flashbacks" & $28(37,3 \%)$ & $21(28 \%)$ & $12(16 \%)$ & $8(10,7 \%)$ & $6(8 \%)$ \\
\hline Malestar psicológico & $11(14,7 \%)$ & $19(25,3 \%)$ & $16(21,3 \%)$ & $17(22,7 \%)$ & $12(16 \%)$ \\
\hline Malestar fisiológico & $30(40 \%)$ & $19(25,3 \%)$ & $11(14,7 \%)$ & $11(14,7 \%)$ & $4(5,3 \%)$ \\
\hline \multicolumn{6}{|l|}{ Evitación } \\
\hline Evitar pensar, sentir... & $19(25,3 \%)$ & $13(17,3 \%)$ & $16(21,3 \%)$ & $11(14,7 \%)$ & $16(21,3 \%)$ \\
\hline Evitar actividades, lugares... & $27(36 \%)$ & $16(21,3 \%)$ & $11(14,7 \%)$ & $10(13,3 \%)$ & $11(14,7 \%)$ \\
\hline Amnesia & $34(45,3 \%)$ & $18(24 \%)$ & $14(18,7 \%)$ & $4(5,3 \%)$ & $5(6,7 \%)$ \\
\hline Pérdida de interés en actividades significativas & $35(46,7 \%)$ & $11(14,7 \%)$ & $11(14,7 \%)$ & $8(10,7 \%)$ & $10(13,3 \%)$ \\
\hline Sensación de desapego o enajenación & $23(30,7 \%)$ & $13(17,3 \%)$ & $20(26,7 \%)$ & $7(9,3 \%)$ & $12(16 \%)$ \\
\hline Restricción de la vida afectiva & $33(44 \%)$ & $11(14,7 \%)$ & $15(20 \%)$ & $8(10,7 \%)$ & $8(10,7 \%)$ \\
\hline Sensación de futuro desolador & $36(48 \%)$ & $14(18,7 \%)$ & $11(14,7 \%)$ & $8(10,7 \%)$ & $6(8 \%)$ \\
\hline \multicolumn{6}{|l|}{ Aumento de la Activación } \\
\hline Insomnio & $25(33,3 \%)$ & $12(16 \%)$ & $15(20 \%)$ & $11(14,7 \%)$ & $12(16 \%)$ \\
\hline Irritabilidad & $21(28 \%)$ & $15(20 \%)$ & $13(17,3 \%)$ & $10(13,3 \%)$ & $16(21,3 \%)$ \\
\hline Dificultades de concentración & $32(42,7 \%)$ & $9(12 \%)$ & $19(25,3 \%)$ & $8(10,7 \%)$ & $7(9,3 \%)$ \\
\hline Hipervigilancia & $24(32 \%)$ & $13(17,3 \%)$ & $19(25,3 \%)$ & $10(13,3 \%)$ & $9(12 \%)$ \\
\hline Respuesta de sobresalto & $19(25,3 \%)$ & $15(20 \%)$ & $18(24 \%)$ & $12(16 \%)$ & $11(14,7 \%)$ \\
\hline
\end{tabular}

presenta sintomatología correspondiente al subsíndrome del mismo, es decir, presencia de uno de los síntomas de reexperimentación (el criterio B tal y como es necesario para el diagnóstico del TEP, según el DSM-IV) y cumplir el criterio $\mathrm{C}$ (al menos tres de los síntomas de evitación) o el criterio D (al menos dos síntomas de incremento de la activación), pero no $\operatorname{ambos}^{17}$.

En la tabla 3 se puede observar las prevalencias obtenidas de cada uno de los síntomas evaluados. Los síntomas más frecuentes observados son el malestar psicológico intenso al recordar algún aspecto relacionado con la situación de abuso $(38,7 \%)$, el evitar pensar, sentir o hablar acerca del suceso (36\%), la irritabilidad $(34,6 \%)$, insomnio y respuestas de sobresalto exageradas (ambos presentes en un $30,7 \%$ de los niños/as y adolescentes).
Los síntomas menos frecuentes son principalmente de evitación: amnesia (12\%), sensación de futuro desolador $(18,7 \%)$, restricción de la vida afectiva $(21,4 \%)$, pérdida de interés en actividades significativas $(24 \%)$ y evitación de actividades lugares o personas que recuerden el suceso (28\%). También destacan los síntomas de reexperimentación: sueños intrusivos y flashbacks (ambos presentes sólo en un $18,7 \%$ de los participantes) y el malestar fisiológico intenso al recordar algún aspecto del trauma (20\%), así como las dificultades de concentración (20\%) dentro de los síntomas de activación.

Se realizaron análisis para determinar la existencia de diferencias entre grupos en cuanto a presencia y ausencia de sintomatología postraumática para identificar posibles variables de riesgo. Se realizaron análisis con 
las siguientes variables: género, edad, tipo de agresión sexual, tipo de agresor, tiempo transcurrido entre la agresión y la aplicación de la escala, si la familia le cree al niño/a o adolescente y si recibe tratamiento tras la agresión. No se encontraron diferencias estadísticamente significativas en ninguna de las comparaciones realizadas.

\section{Discusión}

El desarrollo de estrés postraumático (ya sea sintomatología postraumática o trastorno de estrés postraumático propiamente tal) en niños (as) y adolescentes es uno de los posibles efectos del abuso sexual infantil ${ }^{2}$. El presente estudio confirma la alta prevalencia de esta sintomatología en niños (as) y adolescentes consultantes de centros de peritaje y tratamiento de abuso sexual en nuestra realidad. Un 21,3\% cumple con los criterios para el diagnóstico de TEP, y un $16 \%$ adicional cumple con los criterios de subsíndrome de Estrés Postraumático.

Las cifras señaladas confirman la importancia de evaluar la presencia de sintomatología postraumática en niños (as) y adolescentes que han vivido abuso sexual. La sintomatología postraumática afecta de modo significativo el bienestar y calidad de vida de las personas y tiende en muchos casos a no remitir espontáneamente y requerir un tratamiento específico. Esta sintomatología resulta incapacitante y generadora de malestar permanente, bloqueando los recursos personales para afrontar la situación traumática ${ }^{6,18}$.

En cuanto a la relevancia clínica del subsíndrome de Estrés Postraumático, seguimientos realizados en pacientes que lo presentan han mostrado que éste puede convertirse en TEP en el breve período de un mes y esta posibilidad aumenta significativamente si el período que transcurre es mayor (en torno al año) ${ }^{19}$. Es muy probable que este subsíndrome afecte de forma importante a las víctimas de abusos sexuales como paso previo o desarrollo alternativo al TEP.

El instrumento empleado en la presente investigación no ha sido empleado en estudios equivalentes, por lo que es difícil realizar comparaciones de los resultados obtenidos. Sin embargo, las investigaciones coinciden en indicar que un tercio, sino más, de los niños o adolescentes sexualmente abusados, en muestra clínicas, presentan estrés postraumático.

El presente estudio no evaluó el grado de impedimento asociado a la sintomatología postraumática. En compensación, utilizó un criterio conservador para considerar los síntomas como presentes. Los síntomas informados como presentes "sólo una vez" o "entre dos y tres veces" en las últimas dos semanas no calificaban como presentes para la evaluación de cumplimiento de los criterios diagnósticos de TEP. Este criterio conservador, si bien puede subestimar la presencia de síntomas, permite asumir que los síntomas que fueron reconocidos como tales tienen significación clínica y requieren una consideración cuidadosa.

Es interesante analizar la forma que toma el TEP en este tipo de víctimas: desarrollan con más frecuencia síntomas de aumento de activación que de los otros dos grupos sintomáticos (reexperimentación y evitación). Ello podría explicarse por las características específicas del tipo de trauma sufrido. En general los abusos o violaciones se viven de manera repetida y a manos de un familiar o de una persona conocida, por lo que quizá sea lógico esperar que las conductas de evitación sean menores que en otro tipo de víctimas. Asimismo, la elevada hiperactivación fisiológica puede ser consecuencia de la posibilidad y el temor constante de vivir un nuevo episodio de abuso, ya que no hay claves de seguridad en su propio ambiente. De este modo, la elevada hiperactivación funcionaría como una respuesta adaptativa ante la situación del maltrato.

Es de importancia destacar que si bien el TEP aparece como una de las consecuencias más frecuentes del abuso sexual infantil una parte relevante de la muestra no presentaba TEP propiamente. Ello puede indicar la presencia de procesos protectores en esos niños o adolescentes. Por otro lado, sin duda que el TEP no agota las consecuencias negativas que el abuso sexual puede implicar tanto en las etapas presentes de su desarrollo como etapas futuras.

El carácter transversal de este estudio y el 
tamaño muestral limitan las conclusiones que pueden obtenerse respecto de la identificación de variables de riesgo. Es particularmente de interés que no se observaron diferencias en la prevalencia de estrés postraumático entre quienes estaban en fase de evaluación por su situación de abuso y quienes estaban recibiendo tratamiento. Investigaciones posteriores debieran permitir profundizar en estos aspectos.

\section{Referencias}

1.- Beitchman JH, Zucker KJ, Hood JE, Dacosta GA, Akman D, Cassavia, E: A review of the long-term effects of child sexual abuse. Child Abuse \& Negl 1992; 16 : 101-18.

2.- Putnam F: Ten-year research update review: Child Sexual Abuse. J Am Acad Child Adolesc Psychiatry 2003; 42 (3): 269-78.

3.- Vitriol V, Gomberoff M, Basualto ME, Ballesteros $S$ : Relación entre trastorno por estrés postraumático de inicio tardío, abuso sexual infantil y revictimización sexual. Caso Clínico. Rev Med Chile 2006; 134: 1302-5.

4.- Barudy J: El dolor invisible de la Infancia: Una lectura ecosistémica del maltrato infantil. Barcelona: Paidós; 1998

5.- Kendall-Tackett K, Meyer L, Finkelhor D: Impact of sexual abuse on children: A review and synthesis of recent empirical studies. Psychol Bull 1993; 113 (1): 164-80.

6.- Echeburúa E, Corral P: Secuelas emocionales en víctimas de abuso sexual en la infancia. Cuadernos de Medicina Forense 2006; 12 (43-44): 75-82.

7.- Glaser D: Child sexual abuse. En Rutter M, Bisshop D, Pine D, Scott S, Stevenson J, Taylor E. Rutter's Child and adolescent Psychiatry, Malden: Blackwell, 2008; p. 440-58.

8.- Rind B, Tromovitch P, Bauserman R: A meta-analytic examination of assumed properties of child sexual abuse using college samples. Psychol Bull 1998; 124: 22-53.

9.- Dallam SJ, Gleaves DH, Cepeda-Benito A, Silberg JL, Kraemer HC, Spiegel D: The effects of child sexual abuse: Comment on Rind, Tromovitch, and Bauserman (1998). Psychol Bull 2001; 127: 715-33.

10.- Blanco C, Cabra O, Gómez V, Cassola R, Lapastora P: Consecuencias de la violencia: Patología psiquiátrica asociada al abuso sexual en la infancia. http://www. psiquiatria.com/articulos/trastornos_infantiles/24821/; 2006.

11.- Mcleer S, Deblinger E, Atkins M, Foa E, Ralphe D: Postraumatic stress disorder in sexually abused children. J Am Acad Child \& Adolesc Psychiatry 1988; 27: 650-4.

12.- Trowell J, Ugarte B, Kolvin I, Berelowitz M, Sadowski $J$, Le Couteur A: Behavioural psychopathology of child sexual abuse in school girls referred to a tertiary centre: A North London study. Eur Child Adolesc Psychiatry 1999; 8: 107-16.

13.- Foa E, Johnson K, Feeny N. Treadwell K: The Child PTSD Symptom Scale: A preliminary examination of its psychometric properties (CPSS). J Clin Child Psychol 2001; 30 (3): 376-84.

14.- Bustos P, Rincón PP, Aedo J: Validación preliminar de la escala infantil de síntomas del trastorno de estrés postraumático (Child PTSD Symptom Scale, CPSS) en niños y adolescentes víctimas de violencia sexual. Psykhe 2009; 18 (2): 113-26.

15.- Asociación Psiquiátrica Americana. Manual diagnóstico y Estadístico de los trastornos mentales (4th ed. texto revisado). Washington, DC: APA; 2000.

16.- Echeburúa E, Corral P, Amor P, Zubizarreta I, Sarasua $B$ : Escala de gravedad de síntomas del trastorno de estrés postraumático: Propiedades psicométricas. Análisis y Modificación de Conducta 1997; 23: 503-26.

17.- Hickling EJ, Blanchard EB: The private practice psychologist and manual-based treatments: Post-traumatic stress disorder secondary to motor vehicle accident. Behav Res Ther 1997; 35 (3): 191-203.

18.- Rincón PP, Labrador FJ, Arinero M, Crespo M: Efectos psicopatológicos del maltrato doméstico. Avan Psicol Latinoam 2004; 22: 105-16.

19.- Blanchard EB, Hickling EJ, Barton KA, Taylor AE, Loos $W R$, Jones-Alexander J: One-year prospective followup of motor vehicle accident victims. Behav Res Ther 1996; 34 (10): 775-86. 